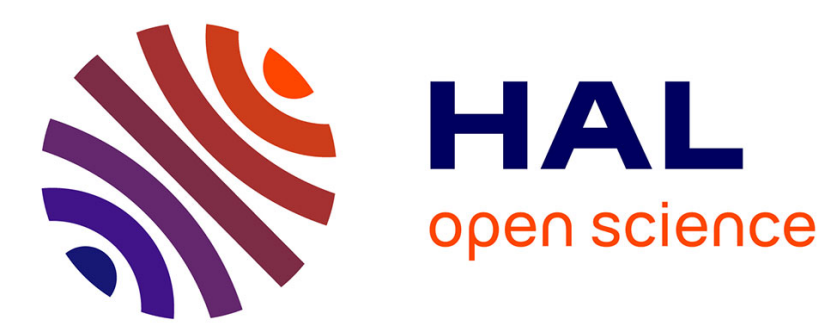

\title{
PROBLEMES ET MODELES EN THEORIE DES SONDES RADIOFREQUENCES
}

\author{
M. Navet, Pierre Bertrand, M. Feix, B. Rooy, L. Storey
}

\section{To cite this version:}

M. Navet, Pierre Bertrand, M. Feix, B. Rooy, L. Storey. PROBLEMES ET MODELES EN THEORIE DES SONDES RADIOFREQUENCES. Journal de Physique Colloques, 1971, 32 (C5), pp.C5b-189-

C5b-191. 10.1051/jphyscol:19715120 . jpa-00214696

\section{HAL Id: jpa-00214696 https://hal.science/jpa-00214696}

Submitted on 1 Jan 1971

HAL is a multi-disciplinary open access archive for the deposit and dissemination of scientific research documents, whether they are published or not. The documents may come from teaching and research institutions in France or abroad, or from public or private research centers.
L'archive ouverte pluridisciplinaire HAL, est destinée au dépôt et à la diffusion de documents scientifiques de niveau recherche, publiés ou non, émanant des établissements d'enseignement et de recherche français ou étrangers, des laboratoires publics ou privés. 
PROBLEMES ET MODELES EN THEORIE DES SONDES RADIOFREQUENCES

\author{
M. Navet - P. Bertrand \\ Groupe Physique Thêorique et Plasma - Université de Nancy I \\ M.R. Feix - B. Rooy - L.R.O. Storey \\ Groupe de Recherches Ionosphériques - Orlêans - La Source
}

\title{
Résumé
}

Les modèles "multiple Water Bag" (N 20) et "Cauchy d'ordre élevé" (N 12) sont des plus utiles pour calculer les impédances propres et mutuelles de dipôles et quadripôles. Cela vient du fait que le calcul des champs à courte distance implique l'utilisation de tous les pôles de la relation de dispersion. De plus, le modèle multiple WB approxime très efficacement une fonction de distribution obtenue expérimentalement.

\section{Abstract}

The multiple (N 20) Water Bag and the high order (N 12) Cauchy models are very useful to compute the mutual and self impedances of dipoles and quadripoles (plane and spherical geometry). This is due to the fact that the computation of fields at short distance implies all the poles of the dispersion relation. Moreover the multiple Water Bag approximates very efficiently an experimentally given distribution function.

\section{I - INTRODUCTION}

Nous nous proposons de calculer les champs électriques créés par des systèmes de grilles planes ou sphériques et d'en déduire les impédances des condensateurs plans et sphêriques formés par deux de ces grilles. Le plasma est homogène, sans collision, sans champ magnétique extérieur, et non relativiste. Les ions peuvent être considêrés comme immobiles. Les grilles sont supposées couplëes électriquement mais non mécaniquement au plasma, ce qui est certainement réalisable au moins pour des plasmas spatiaux.

Le calcul des champs électriques nécessite la connaissance de 1 a constante diélectrique longitudinale du plasma. Celle-ci peut se déduire de la fonction de distribution des vitesses $F(\vec{V})$ des électrons (normalisée à l quand on intègre sur 1 'espace des vitesses) :

$$
\text { (1) } \varepsilon(\vec{k}, \omega)=1+\frac{\omega^{2} p}{k^{2}} \frac{\vec{k} \cdot \partial F(\vec{v}) / \partial \vec{v}}{\omega-\vec{k} \cdot \vec{v}} d^{3} v
$$

oĩ $\omega$ est la pulsation, $\omega_{p}$ la pulsation de plasma, $\vec{k}$ le vecteur nombre d'onde. [1]

Cependant, le calcul de $\varepsilon(\vec{k}, \omega)$ et des racines de $E(\vec{k}, \omega)=0$ pour une pulsation donnée, ou pour un vecteur nombre d'onde $\vec{k}$ donné, $n$ 'est pas aussi simple qu'il semble à première vue et des difficultés à la fois théoriques et numériques apparaissent. Aussi, avant d'étudier les problèmes pratiques de la détermination des champs électriques et des impê- dances, nous allons passer rapidement en revue 5 modèles. Ensuite, nous adopterons $1^{\prime}$ un de ces modèles - le supercauchy- come base de la discussion des problèmes ci-dessus.

\section{II - LES MODELES}

\section{1. - Modèle du plasma froid}

(2) $F(\vec{V})=\delta(\vec{v})$ où $\delta$ dêsigne la distribution de Dirac. On en déduit :

(3) $\varepsilon(\vec{k}, \omega)=1-\omega_{p}^{2} / \omega^{2}$

E est indépendant du nombre d'onde $k$. Le défaut du modèle est que c'est un cas limite où le mouvement thermique est totalement absent.

\subsection{Modèle Maxwellien}

Dans ce modèle la fonction de distribution des vitesses est :

(4) $\quad F(\vec{V})=(2 \pi)^{-{ }_{3} / 2} V_{T}^{-3} \exp \left(-V^{2} / 2 V_{T}^{2}\right)$

où $V_{T}=(K T / m)^{1 / 2}$, avec $K$ la constante de

Boltzmann, m la masse de l'électron, T la température. On obtient alors :

$$
\text { (5) } \epsilon(k, \omega)=1-\frac{1}{2} \frac{\omega_{P}^{2}}{k^{2} V_{T}^{2}} \quad z\left(\frac{\omega}{k V_{T} \sqrt{2}}\right)
$$

où $Z$ est la fonction de FRIED et CONTE [2]. Le nombre des racines de 1 'équation de dispersion est infini et des difficultés thêoriques de convergence peuvent se présenter. DENAVIT [3] montre par exemple que dans le problème des oscillations libres, 1a 
série :

(6) $\sum_{j=1}^{\infty} B_{j} \exp \left(i \omega_{j} t\right)$ dans laquelle les $B_{j}$ sont les facteurs d'excitation associés aux racines $\omega_{j}$ de l'équation de dispersion pour un $k$ réel donné, ne converge pas uniformément lorsque $t$ tend vers 0 . On peut s'attendre à des difficultés plus graves dans le problème des oscillations forcées.

\section{3. - Modè le "Supercauchy"}

Ces difficultés mathématiques nous ont conduits à envisager de nouveaux modèles, dont le premier, appelé "Supercauchy" [4] utilise le fait que la

(7) $F_{n}(V)=\frac{\Gamma(n)}{V_{T}^{3} \pi^{3 / 2}(2 n-5)^{3 / 2} \Gamma\left(n-\frac{3}{2}\right)}\left(1+\frac{V^{2}}{(2 n-5) V_{T}^{2}}\right)^{-n}$

tend pour $\mathrm{n}$ suffisamment grand vers 1a distribution de Maxwell dont, à partir de $n=12$, elle ne diffère que pour les particules très suprathermiques qui, de toute manière, sont en nombre négligeable. Le problème est mathématiquement relativement simple. L'équation de dispersion a une forme polynomiale, possêde $n$ racines et nous avons un traitement mathématique complet. Toutefois, à très grande distance de la source, seules les particules à très grande vitesse contribuent au champ et ce modèle ne donne pas la forme asymptotique correcte. Remarquons que cette forme n'est valable que pour des champs très faibles, et que pour nos problèmes il est plus intéressant d'avoir des formules valables à distance finie. Pratiquement des études numériques [5] ont montré que pour $n=12$ les résultats pour le champ sont valables, à titre d'approximation du champ qui s'obtient avec la distribution maxwellienne, pour des distances allant jusqu'à 60 longueurs de Debye.

\section{4. - Modèle du "Water Bag"}

Le "Water Bag" a étê utilisê par GRARD [6] dans des calculs de sondes quadripolaires. II $s$ 'agit de la fonction de distribution (8) $\quad \mathrm{F}(\overrightarrow{\mathrm{v}})=\frac{1}{4 \pi a^{2}} \delta(\mathrm{V}-\mathrm{a})$ où $\mathrm{V}=|\overrightarrow{\mathrm{v}}|$. Projetée sur I'axe des $\mathrm{x}$ cette distribution s'êcrit :

(9) $\quad F\left(V_{x}\right)=\frac{1}{2 a} H\left(V_{x}+a\right)-H\left(V_{x}-a\right)$

(10) $H(u)=0$ si $u<0$

$$
\text { l si } u>0
$$

Dans ces conditions, on obtient :

(11) $\varepsilon(\vec{k}, \omega)=1-\frac{\omega_{p}^{2}}{\omega^{2}-k^{2} a^{2}} \quad$ Pour un $\omega$ donné 1 'équation de dispersion n'a que 2 racines en $k$.
Ce modèle a pour principal défaut de ne pas comporter le phénomène d'amortissement Landau.

\section{5. - Modèle du "Multiple Water Bag"}

Récemment [7] le modèle du Water Bag a été généralisé avec une fonction de distribution pour chaque composante axiale de la vitesse donnée par $F\left(V_{x}\right)=\sum_{j=1}^{N} A_{j}\left[H\left(V_{x}+a_{j}\right)-H\left(V_{x}-a_{j}\right)\right]$

avec la condition de normalisation :

(13) $2 \sum_{j=1}^{N} a_{j} A_{j}=1:$ Il s'agit de la superposition de $N$ "bags" chacun ayant la hauteur $A_{j}$. Ce modèle permet d'approximer toute fonction de distribution à symétrie sphêrique, c'est-à-dire isotrope. Il est même particulièrement adapté au cas oũ $1^{\text {'on }}$ connait la fonction de distribution seulement sous forme de tableau numérique. On peut dire que les "bags" constituent alors les briques élémentaires avec lesquelles on peut approximer $F(\vec{V})$, (qui, avec des $A_{j}$ négatifs, peut très bien ne pas être monotone dếcroissante en $|\vec{v}|)$. La constante diêlectrique s'écrit :

(14) $\varepsilon(\vec{k}, \omega)=1+\omega_{p}^{2} \sum_{j=1}^{N} \frac{2 a_{j} A_{j}}{k^{2} a_{j}^{2}-\omega^{2}}$

Pour une pulsation wdonnée, I'équation de dispersion a $2 \mathrm{~N}$ racines réelles. Mais 1 'amortissement Landau apparait sous forme d'un mélange de phase "à la Van Kampen"; il subsiste pendant un temps (dans un problème à oscillations libres) ou sur une distance (dans un problème à oscillations forcées) qui est d'autant plus long que $N$ est plus grand. Parmi les nombreux avantages du modèle multiple Water Bag, signalons la simplicité du calcul des racines de 1 'équation de dispersion pour $k$ donné. Par exemple, si tous les $A_{j}$ sont positifs, la racine $\omega_{i}$ est telle que : $k a_{i}<\omega_{i}<k a_{i+1}$. En conclusion, ce modèle se révèle des plus utilestant sur le plan théorique que sur le plan numérique.

\section{III - LES PROBLEMES}

\section{1. - Différentes géométries}

Nous allons comparer les champs créés, d'une part par une grille plane de densitê de charge superficielle $\sigma \cos \omega t$, et d'autre part, par une charge ponctuelle q cos $\omega t$. Leur calcul nêcessite une intégration dans $l$ 'espace des $\vec{k}$. Dans les deux cas, $I^{\prime}$ expression de $\varepsilon(\vec{k}, \omega)$ est la même et la seule différence provient des poids différents dans 1 'espace des $\vec{k}$. Tous les calculs faits il apparait 
que le champ en géométrie plane, et le potentiel en géométrie sphérique, sont donnēs par des expressions analogues. Supercauchy donne :

(15)en géométrie plane $E(x)={ }_{2 E_{0}}^{\sigma}\left[\frac{1}{E_{f}}+\frac{2}{\pi} \sum_{j=1}^{N} G_{j} \vec{F}^{\prime}\left(k_{j} x\right)\right]$

(16) en géométrie sphérique :

$v_{\omega}(r)=\frac{9}{4 \pi E_{0}}\left[\frac{1}{E_{f}}+\frac{2}{\pi} \sum_{j=1}^{N} B_{j} F\left(K_{j} r\right)\right.$

où (17) $\mathcal{F}_{(u)}^{0}=\int_{0}^{\infty} \frac{\sin t}{t-u} d t$

$\varepsilon_{f}$ est la constante diélectrique d'un plasma froid de même densité et les $B_{j}$ les coefficients d'excitation associés aux racines $k_{j}$ de $l$ 'équation de dispersion.

\section{2. - Etude de certains cas limites}

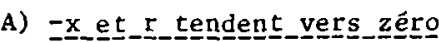

Dans ces conditions, on a $(0)=\pi / 2$. Comme il est aisé de montrer que : (18) $\varepsilon_{f}^{-1}+\sum_{j=1}^{n} B_{j}=1$ On voit que (19) $E_{\omega}(x)=\frac{\sigma}{\text { (20) } v_{\omega}(r)=\frac{q}{4 \pi \varepsilon_{0} r}}$ On retrouve les formules du vide ce qui correspond bien au fait que dans un plasma chaud l'effet de polarisation ne joue qu'au-delà d'une certaine distance, qui varie avec la fréquence.

\section{B) _-_x_et}

Dans ces conditions, $\vec{F}_{(u)}$ tend vers 0 et l'on retrouve le résultat du modèle plasma froid, soit le champ ou le potentiel du vide divisé par $\varepsilon_{f}$. c) _- $\omega$ tend_vers $\omega$

Il est alors intéressant de remarquer que le terme plasma froid et la contribution des pôles de Landau $(j=1,2)$ se détruisent partiellement. Les formules

(15) et (16) deviennent respectivement :

$$
\begin{aligned}
& \text { (21) } E\left(x, \omega+\omega_{p}\right)={ }_{2 \varepsilon_{0}}^{\sigma}\left[\frac{i x}{D \sqrt{3 \varepsilon_{f}}}+\alpha+\frac{x^{2}}{6 D^{2}}+\frac{2}{\pi} \sum_{j=3}^{n} B_{j} F\left(k_{j} x\right)\right] \\
& \text { (22) } V\left(r, \omega+\omega_{p}\right)={ }_{4 \pi \varepsilon_{0}}^{q}\left[\frac{i}{D \sqrt{3 \varepsilon_{f}}}+\frac{\alpha}{r}+\frac{r}{6 D^{2}}+\frac{2}{\pi} \sum_{j=3}^{n} B_{j} F^{\prime}\left(k_{j} r\right)\right]
\end{aligned}
$$

où $\alpha$ est un facteur numérique qui tend vers $5 / 3$ lorsque $n$ tend vers l'infini. Dans les membres de droite, le ler des termes entre parenthèses tend vers l'infini lorsque $\omega$ tend vers $\omega_{p}$, car $\varepsilon_{f}$ tend alors vers 0 ; les autres termes restent finis. (21) et (22) montrent une différence intêressante entre un système plan et un système sphérique dans le cas d'une excitation dipôlaire: le terme dominant est bien un terme dipôlaire dans le cas d'une géométrie plane (c-à-d proportionnel à $\sigma \Delta$, où $\Delta$ est la distance entre les 2 grilles), alors qu'en géomêtrie sphériniso le terme correspondant $s^{\prime a n-}$ nule.

3.3. - Constantes diélectriques apparentes de condensateurs à grilles en géométries plane et sphérique : $\varepsilon_{p}$ et $\varepsilon_{s}$ $\frac{\text { Géomêtrie plane }}{(23)}: \frac{1}{\varepsilon_{p}(\omega)}=\frac{\Delta}{2 \pi}\left[\left[\frac{\sin (k \Delta / 2)}{k \Delta / 2}\right]^{\infty} \frac{1}{\varepsilon(k, \omega)} d k\right.$

(24) $=\frac{1}{\varepsilon_{f}}+\frac{2}{\pi} \sum_{j=1}^{n} B_{j} \tilde{F}^{H}\left(k_{j} \Delta / 2\right)$ avec (25) $\tilde{F}^{1}(u)=\int_{0}^{\infty} \frac{\sin ^{2} t}{t(t-u)} d t$

En particulier, dans le cas limite où $\omega$ tend vers

$$
\omega_{p} \text {, on a (26) } \frac{1}{\varepsilon_{p}\left(\Delta, \omega \rightarrow \omega_{p}\right)}=i \frac{\Delta / D}{2 \sqrt{3 \varepsilon_{f}}}
$$

de sorte que $\varepsilon_{p}$ tend vers 0 lorsque $\omega$ tend vers $\omega_{\mathrm{p}}$.

Géométrie sphérique : Comme nous l'avons déjà dit, le cas sphérique est très différent. Si 1 'on considère 2 sphères concentriques de rayons $a$ et $b$, on peut d'abord obtenir la formule générale :

(27)

$$
\begin{aligned}
& \frac{1}{\varepsilon_{s}(a, b)}=\frac{a}{b-a} \frac{1}{\varepsilon_{p}(2 b)}+\frac{b}{b-a} \frac{1}{\varepsilon_{p}(2 a)} \\
& -\frac{b+a}{b-a} \frac{1}{\varepsilon_{p}(b-a)}+\frac{1}{\varepsilon_{p}(b-a)}
\end{aligned}
$$

Si l'on tient compte de la forme asymptotique (26) on démontre que lorsque $\omega$ tend vers $\omega_{p}, \varepsilon_{s}$ ne tend pas vers zéro, mais vers une limite finie.

Ce genre d'excitation, présente donc l'interressante particularité de mettre en jeu les modes électrostatiques au delà des pôles principaux de Landau, et la forme des courbes de réponse doit permettre de préciser les paramètres du plasma.

\section{BIBLIOGRAPHIE}

[I] BEKEFI (G.), "Radiation processus in plasmas" Wiley, New York, 1966.

[2] FRIED (B.D.) et CONTE (S.D.), "The plasma dispersion function", Academic Press, New York, 1961.

[3] Denavit (J.), Phys. Fluids., 1968, II, 680.

[4] FEIX (M.R.), ROOY (B.), C.R.A.S., 1970, 270, 148.

[5] ROOY (B.), "Théorie de la sonde quadripolaire en plasma chaud isotrope", Plasma Phys. à par.

(6) GRARD (R.J.I.), Alta Frequenza, 1969, 38, 97.

[7] NAVET (M.) BERTRAND (P.), Phys.Let.1971,34A, 117. 